\title{
Effectiveness of work-related medical rehabilitation in cancer patients: study protocol of a cluster-randomized multicenter trial
}

\author{
Julian Wienert ${ }^{*} \mathbb{D}$, Betje Schwarz and Matthias Bethge
}

\begin{abstract}
Background: Work is a central resource for cancer survivors as it not only provides income but also impacts health and quality of life. Additionally, work helps survivors to cope with the perceived critical life event. The German Pension Insurance provides medical rehabilitation for working-age patients with chronic diseases to improve and restore their work ability, and support returning to or staying at work, and thus tries to sustainably avoid healthrelated early retirement. Past research showed that conventional medical rehabilitation programs do not support returning to work sufficiently and that work-related medical rehabilitation programs report higher return-to-work rates across several health conditions, when compared to medical rehabilitation. Therefore, the current study protocol outlines an effectiveness study of such a program for cancer survivors.
\end{abstract}

Methods: To evaluate the effectiveness of work-related medical rehabilitation in cancer patients we conduct a cluster-randomized multicenter trial. In total, 504 rehabilitation patients between 18 and 60 years with a Karnofsky Performance Status of $\geq 70 \%$, a preliminary positive social-medical prognosis of employability for at least $3 \mathrm{~h} /$ day within the next 6 months and an elevated risk of not returning to work will be recruited in four inpatient rehabilitation centers. Patients are randomized to the work-related medical rehabilitation program or the conventional medical rehabilitation program based on their week of arrival at each rehabilitation center. The work-related medical rehabilitation program comprises additional work-related diagnostics, multi-professional team meetings, an introductory session as well as work-related functional capacity training, work-related psychological groups, and social counseling. All additional components are aimed at the adjustment of the patients' capacity in relation to their individual job demands. Role functioning defines the main study outcome and will be assessed with the EORTC-QLQ30. Secondary outcome measures are the remaining scales of the EORTC-QLQ30, fatigue, self-rated work ability, disease coping, participation in working life, realization of work-related goals and therapies during rehabilitation, and treatment satisfaction.

Discussion: A positive evaluation of work-related medical rehabilitation in cancer patients is expected due to the promising findings on the effectiveness of such programs for patients with other health conditions. Results may support the dissemination of work-related medical rehabilitation programs in German cancer rehabilitation.

Trial registration: German Clinical Trials Register DRKS00007770. Registered 13 May 2015.

Keywords: Cancer, Medical rehabilitation, Work-related medical rehabilitation, Work-related therapies, Return to work, Inclusion, Cluster-randomized trial, Study protocol

\footnotetext{
* Correspondence: julian.wienert@uksh.de

Institute for Social Medicine and Epidemiology, University of Lübeck,

Ratzeburger Allee 160, 23562 Lübeck, Germany
} 


\section{Background}

With approximately 14.1 million new cases in 2014, cancer is one of the leading causes of morbidity worldwide [1]. Europe alone accounts for $24.4 \%$ (approximately 3.44 million cases) of the worldwide incidence. The prostate (22.8\%), lung (15.9\%), and colorectum (13.2\%) are the most prominent cancer sites among men in Europe. Among women, the breast (28.8\%), colorectum (12.7\%), and lung $(7.4 \%)$ are the most frequently affected sites [1]. These numbers also mirror the current situation in Germany where 477,300 incident cases were reported for 2010 [2].

Due to better screening and treatment of cancer, survival rates in cancer patients have improved steadily over the last few years in most developed countries. Five-year survival rates for cancer improved for patients diagnosed between 2005 and 2009 in most developed countries when compared to 5-year survival rates between 1995 and 1999 (e.g. Germany: 48.7 to $64.6 \%$ for colon cancer, 51.9 to $62.1 \%$ for rectal cancer, 81.2 to $85.3 \%$ for breast cancer) [3]. As a result, the life situation of cancer survivors and their participation in different life domains received increasing attention from patients, clinicians, and researchers [4]. One of these important life domains is work. Work not only provides income to afford living, it also helps to structure time, enables social contacts, and develops skills and personality [5, 6]. The presence or absence of work has an impact on various facets of health and quality of life $[7,8]$. Work also has a positive influence on coping with a disease (e.g. stroke [9, 10]; psychiatric disorders [11]; multiple sclerosis [12]). For cancer survivors, returning to work after cancer diagnosis and subsequent treatment may support their ability to cope with this critical life event and represents a major step in restoring normality [13-16].

Although up to $75 \%$ of cancer survivors previously working in a job return to it after treatment [17], returning to work is often easier said than done. Cancer survivors are 1.4 times more likely to be unemployed compared to healthy controls [18]. They also have an increased risk of early retirement and are less likely to be re-employed [18, 19]. Reviews showed that a multitude of parameters influence the process of returning to work in cancer survivors, including health, well-being and symptoms, and different domains of functioning, as well as self-rated work ability and work-related factors such as job demands and the working environment [18-22]. In line with general return-to-work (RTW) research [23], RTW in cancer survivors can therefore be described as a multifaceted bio-psycho-socially moderated process. Consequently, comprehensive multidisciplinary rehabilitation strategies are favored for supporting RTW. A Cochrane review by de Boer et al. [19] confirmed that only diverse, multidisciplinary interventions show moderate evidence of higher RTW rates for cancer survivors when compared to usual care (for a comprehensive review across several health conditions, please see Hoefsmit et al. [24]). Established programs, however, are not necessarily multidisciplinary and often lack a specific focus on RTW [25]. In Germany, the German Pension Insurance (GPI) provides medical rehabilitation (MR) for working-age patients with chronic diseases to improve and restore their work ability, and support returning to or staying at work, and thus tries to sustainably avoid health-related early retirement. In Germany, about one fifth of all rehabilitation measures are provided due to cancer [26]. However, though improvement and restoration of work ability are the primary aims of German MR programs, conventional MR does not seem to address work-related issues sufficiently [27, 28]. The evidence considering the effects of these programs on work-related outcome measures in cancer patients is mixed at best. Most studies that are available did not use a control group and focused only on generic health-related outcome measures. These studies showed that MR can increase health-related quality of life, physical functioning, and general mental well-being [29-32], and decrease anxiety and depression [33]. Several studies, however, showed that, in particular, persons with a poor self-rated RTW prognosis did not benefit from conventional MR [34, 35]. Moreover, Weis and colleagues [32], who investigated the work-related impact of inpatient rehabilitation in female breast cancer patients in a controlled study, found no sustainable effects on health- or work-related outcomes [36]. Obviously, conventional MR as practiced in Germany is not a sufficient treatment to address the multifaceted factors of RTW in cancer survivors.

As a result, rehabilitation programs with a stronger focus on work, work ability, and RTW have been developed in recent years. By addressing the individual job demands and necessary skills to meet them, these programs aim to increase the chances of a sustainable participation in the labor market, especially for patients with more severe restrictions of their work ability (e.g. long or repeated periods of sick leave, unemployment, poor self-rated RTW prognosis). To provide a framework for these diverse programs and activities, the GPI published guidelines for the implementation of work-related medical rehabilitation (WMR) [37]. These guidelines define the target group, describe specific work-related diagnostic measures, and explain the core therapeutic modules that complement WMR programs as compared to conventional MR. The definition of the target group, i.e. patients on longterm sick leave and patients with a poor self-rated RTW prognosis, also includes recommendations for the identification of these patients by naming several screening instruments that assess an elevated risk of permanent work disability. The diagnostic process in WMR is described as demand-oriented, meaning that it should assess physical and psycho-social work 
ability against the background of the individual workrelated demands. This also includes the identification of individual and environmental risk factors and resources that might be related to coping with these demands. Additionally, a functional capacity evaluation should be part of the diagnostic procedure [38, 39]. Finally, the core therapeutic modules of WMR as described by the guidelines are a) work-related functional capacity training, b) work-related psychological groups, and c) intensified social counseling [37].

Randomized controlled trials in patients with musculoskeletal [40, 41], cardiac [42], neurological [43], and psychosomatic disorders [44, 45] showed that patients who received WMR reported significantly higher RTW rates than patients who received a conventional MR program. Though WMR is well established for several disorders, there are currently only a few WMR programs for cancer patients. One non-randomized clinical trial in cancer patients showed no differences in RTW rates, work-related stress, and job satisfaction between a WMR group and a conventional MR group [46]. A major methodological shortcoming of this study was the imbalance of both groups regarding several baseline measures. Moreover, the description of the program indicates that it was not a comparably comprehensive strategy as described in the current guidelines for WMR. Therefore, we designed a study to determine the effects of wellimplemented WMR programs on work-related and quality-of-life outcomes in cancer survivors.

\section{Methods}

\section{Design}

The study is designed as a cluster-randomized multicenter trial which is realized in four German rehabilitation centers: the Klinik Bavaria in Freyung, the ParacelsusKlinik am See in Bad Gandersheim, the MediClin Rose Klinik Horn-Bad Meinberg, and the Röpersbergklinik in Ratzeburg. The control group receives conventional MR as prescribed by the German Pension Insurance, whereas the intervention group receives a WMR program. Patients are randomized in clusters. These clusters are defined by a shared start of the rehabilitation program. Randomization is stratified by centers.

\section{Recruitment}

Patients are informed about the study by a study nurse or a physician in charge and have to provide written informed consent prior to study participation. Participants can withdraw their consent at any time without any consequences. No modifications to the intervention are planned. All rehabilitation centers were provided with a process description for recruitment prior to the study start and follow the same procedure.
When giving informed consent to participate in the study, the name and address will be listed by the rehabilitation center and a study number will be assigned. This study number will be entered in a field on each questionnaire. The study number will be also added to the medical discharge letter while all information on name and address will be removed to ensure that research data and confidential data cannot be linked. The study list with personal data and the study number will be destructed at the end of the study $(12 / 31 / 2017)$ by each rehabilitation center to ensure that names and addresses of study participants are no longer available.

The researchers receive a copy of this study list only for mailing the questionnaires 3 and 12 months after discharge. Use of these data is not allowed for any other purpose but sending out the questionnaires. The study list will be secured with a password, stored separately from the collected research data, and erased after sending out the last questionnaires. The time schedule of enrolment and interventions is displayed in Table 1.

\section{Treatment \\ Control}

Participants in the control group receive a conventional MR as provided by rehabilitation centers in Germany. Conventional MR is usually a 3-week program with an overall amount of 60-75 h of therapy [26]. This includes exercise therapy, physiotherapy, social counseling, occupational therapy, psychological seminars and counseling, and dieting. Participants in the control group do not receive intensified work-related therapies. However, social counseling as an obligatory part of conventional MR informs on social law and provides basic knowledge about the rights of persons with disabilities. This also includes work-related issues.

\section{Intervention}

Participants in the intervention group receive a WMR program. In addition to the conventional MR, the WMR includes additional work-related therapies as defined by the WMR guideline (see above) [37]. This guideline prescribes at least 11-25 h of additional work-related content for WMR patients [37]. The complete treatment dose of a WMR program contains up to $100 \mathrm{~h}$ of therapy.

The intervention content is guided by the WMR guidelines [37]. Further specifications of the diagnostic and therapeutic procedures are achieved in collaboration with the rehabilitation centers, based on an implementation phase before the start of the initial trial. This implementation phase is aimed at increasing the feasibility and fidelity of therapies across all participating rehabilitation centers. This phase is also used to check whether centers prescribe WMR therapies in addition to conventional MR therapies for the intervention groups and that control 
Table 1 Time schedule of enrolment and interventions

\begin{tabular}{|c|c|c|c|c|c|c|c|}
\hline \multirow[b]{3}{*}{ Time point } & \multicolumn{7}{|l|}{ Study period } \\
\hline & \multirow{2}{*}{$\begin{array}{l}\text { Enrolment } \\
\text { Pre-admission }\end{array}$} & \multirow[t]{2}{*}{ Allocation } & \multicolumn{4}{|c|}{ Post-allocation } & \multirow{2}{*}{$\begin{array}{l}\text { Close-out } \\
\text { 12-month } \\
\text { follow-up }\end{array}$} \\
\hline & & & Admission & Discharge & $\begin{array}{l}\text { 3-month } \\
\text { follow-up }\end{array}$ & $\begin{array}{l}\text { 12-month } \\
\text { follow-up }\end{array}$ & \\
\hline \multicolumn{8}{|l|}{ Enrolment: } \\
\hline Eligibility screen & $x$ & & & & & & \\
\hline Informed consent & $x$ & & & & & & \\
\hline Allocation & & $x$ & & & & & \\
\hline \multicolumn{8}{|l|}{ Interventions: } \\
\hline Work-related medical rehabilitation & & & $\hookleftarrow$ & & & & $x$ \\
\hline Conventional medical rehabilitation & & & & & & & $x$ \\
\hline
\end{tabular}

groups only receive conventional MR. The additional content of WMR and work-specific therapies as well as their minimum amount of time are described below (see Table 2 for a brief overview).

Additional work-related diagnostics Additional workrelated diagnostics are carried out by the physician in charge, a psychologist and an occupational therapist or physiotherapist directly after admission of the patients. The diagnostic procedures are aimed at a comprehensive assessment of work functioning. Work functioning is understood as the result of the interaction between the health problem on the one hand and work-related environmental and personal factors on the other. Restrictions in work functioning can be related to body functions and structures, activities, and participation [47, 48]. Each of the three involved professions makes its own specific contribution to the comprehensive assessment. The physician primarily focuses on body functions and structures (e.g. impairments). The psychological assessment, comprising interview techniques as well as standardized instruments, focuses on environmental (e.g. support of co-workers and supervisor) and personal (e.g. coping styles) factors that impact work functioning positively as resources and skills or negatively as barriers. Finally, the occupational therapist or physiotherapist assesses the ability to perform work activities by using standardized tests of functional capacity evaluation or structured observations of non-standardized job tasks $[38,39]$. The complete diagnostic process takes at least $60 \mathrm{~min}$. The medical and the psychological assessment take at least 15 min each. The assessment of workrelated functional capacity takes at least $30 \mathrm{~min}$. On the basis of the diagnostic findings, the multi-professional team jointly develops a comprehensive rehabilitation plan with a tailored treatment strategy for each patient.

Multi-professional team meetings Multi-professional team meetings are carried out once a week by the WMR team, i.e. by the physician in charge, the psychologist, the social worker, and at least one occupational therapist and physiotherapist. The meetings provide a platform for mutual exchange about the single patients assigned to the WMR program (case conference). Besides facilitating and bundling communication about the patients, the meetings also aim to coordinate the single WMR elements provided by the different team members to achieve concerted action. The first meeting takes place directly after the aforementioned diagnostic procedures to jointly develop a rehabilitation plan and treatment strategy for each patient, focusing on the enhancement of work functioning. In the second meeting all team members report on the patients' progress and current situation, assessed or observed during or alongside the different therapeutic interventions. If necessary, rehabilitation plans and treatment strategies are adjusted. In the last meeting the individual rehabilitation outcomes are evaluated. Based on this evaluation the final decision on return to work or prolonged sick leave is discussed within the team. Moreover, additional measures and benefits to support return to work are talked about; if needed, recommendations are given to the GPI. Each team meeting takes at least $30 \mathrm{~min}$.

Introductory session The introductory session informs all patients who participate in the WMR program about the structure, content, and aims of the program. Each therapist is introduced with their field of expertise so patients can identify the right person to address expertiserelated questions. The session is organized as a group presentation, which is held by a physician at the beginning of the WMR program and takes a minimum of $30 \mathrm{~min}$. The session is aimed at 1 ) getting patients familiar with the program right from the beginning of their treatment, 2) initiating a treatment collaboration between patients and therapists (i.e. a respectful and trustful relationship between patient and therapist) by providing a transparent structure, and 3) motivating patients to actively participate 
Table 2 Brief overview of the WMR program

\begin{tabular}{|c|c|c|c|c|}
\hline Module & Time & Setting & Professions & Content \\
\hline $\begin{array}{l}\text { Additional work-related } \\
\text { diagnostics }\end{array}$ & $\begin{array}{l}\text { During admission to } \\
\text { rehabilitation } \\
\text { Total: at least } \\
60 \text { minutes } \\
\text { Medical assessment: } \\
15 \text { minutes } \\
\text { Psychological assessment: } \\
15 \text { minutes } \\
\text { Test of functional capacity } \\
\text { and performance: } 30 \text { minutes }\end{array}$ & $\begin{array}{l}\text { Personal } \\
\text { interaction } \\
\text { with the patient }\end{array}$ & $\begin{array}{l}\text { Physician } \\
\text { Psychologist, } \\
\text { psychotherapist } \\
\text { Occupational } \\
\text { therapist, } \\
\text { physiotherapist }\end{array}$ & $\begin{array}{l}\text { Assessment of work functioning and its restrictions } \\
\text { related to body functions and structures as well as } \\
\text { activities and participation } \\
\text { Assessment of environmental and personal factors } \\
\text { that impact work functioning positively as resources } \\
\text { and skills or negatively as barriers } \\
\text { Use of structured patient interviews and/or standardized } \\
\text { assessment instruments as well as standardized tests of } \\
\text { functional capacity evaluation, structured observations } \\
\text { of non-standardized job tasks }\end{array}$ \\
\hline $\begin{array}{l}\text { Multi-professional } \\
\text { team meetings }\end{array}$ & $\begin{array}{l}\text { After admission } \\
\text { During the course } \\
\text { of rehabilitation } \\
\text { Before discharge }\end{array}$ & $\mathrm{N} / \mathrm{A}$ & $\begin{array}{l}\text { All professions } \\
\text { associated with } \\
\text { the WMR program }\end{array}$ & $\begin{array}{l}\text { Individual case conference for each patient } \\
\text { After admission: results of the work-related diagnostics, } \\
\text { joint development of a treatment plan } \\
\text { During the course of rehabilitation: development of the } \\
\text { patient, adjustment of the treatment plan if needed } \\
\text { Before discharge: evaluation of rehabilitation outcomes, } \\
\text { return-to-work prognosis, additional measures to } \\
\text { support return to work if needed }\end{array}$ \\
\hline Introductory session & After admission & $\begin{array}{l}\text { Presentation in } \\
\text { front of all WMR } \\
\text { patients }\end{array}$ & Physician & $\begin{array}{l}\text { Description of the aims of WMR, explanation of the } \\
\text { program structure and each module, introduction of } \\
\text { the rehabilitation team } \\
\text { Inform, motivate and prepare the patient for the } \\
\text { subsequent program } \\
\text { Establish treatment collaboration }\end{array}$ \\
\hline $\begin{array}{l}\text { Work-related functional } \\
\text { capacity training }\end{array}$ & At least 360 minutes & $\begin{array}{l}\text { Training in } \\
\text { small groups } \\
\text { or personal } \\
\text { training }\end{array}$ & $\begin{array}{l}\text { Occupational } \\
\text { therapist, } \\
\text { physiotherapist }\end{array}$ & $\begin{array}{l}\text { Complex and multidimensional tasks to simulate } \\
\text { realistic work-related demands and situations } \\
\text { Integrated ergonomic and cognitive training }\end{array}$ \\
\hline $\begin{array}{l}\text { Work-related } \\
\text { psychological groups }\end{array}$ & At least 240 minutes & $\begin{array}{l}\text { Seminars with } \\
\text { small groups }\end{array}$ & $\begin{array}{l}\text { Psychologist, } \\
\text { psychotherapist }\end{array}$ & $\begin{array}{l}\text { Seminars focusing on work-related stress and coping, } \\
\text { work-related social competencies in communication, } \\
\text { and planning the concrete return to work }\end{array}$ \\
\hline $\begin{array}{l}\text { Intensified social } \\
\text { counseling }\end{array}$ & $\begin{array}{l}\text { At least } 90 \text { minutes: } \\
60 \text { minutes seminars } \\
30 \text { minutes personal } \\
\text { counseling }\end{array}$ & $\begin{array}{l}\text { Seminars with } \\
\text { small groups } \\
\text { or personal } \\
\text { counseling }\end{array}$ & Social worker & $\begin{array}{l}\text { Clarification of the problematic work-related situations } \\
\text { and perspectives, information and consultation on } \\
\text { social law-related topics as well as measures and } \\
\text { benefits to support return to work, establish contact } \\
\text { with employer and request further measures and } \\
\text { benefits from the social or health services agencies } \\
\text { if needed }\end{array}$ \\
\hline
\end{tabular}

in their therapy by providing additional information about the potential benefits of the program.

Work-related functional capacity training Work-related functional capacity training is carried out by an occupational therapist or a physiotherapist in group or personal sessions. Based on diagnostic results and following principles of graded activity (e.g. [49]), an individualized training plan is developed for each WMR patient to purposively increase work-related capacity and facilitate work-related performance. Graded activity comprises regular training with general exercises, strengthening exercises, and tailored exercises that imitate individually relevant physical tasks by making use of operant-conditioning behavioral principles with the aim of restoring functioning. The primary aim is not pain relief. However, participants learn that despite pain symptoms, exercise and physical activity are safe and recommended [49]. Following these principles, training plans not only contain single or one-dimensional motion tasks (e.g. latissimus pull-down exercise), but also complex and multidimensional tasks (e.g. lifting and carrying bricks, tightening bolts overhead, filling a goods shelf, patient transfer) to simulate realistic work-related demands and situations. Simultaneously, therapists train patients in ergonomic working by implementing ergonomics in the regular training (e.g. ergonomic corrections when lifting goods onto a shelf).

Since cognitive impairments (as a symptom, result, or side effect of the disease or its treatment) are often reported by cancer survivors, this module also comprises cognitive training sessions. Amongst others, computer-assisted training or group sessions under the guidance of a therapist are used to improve attention, concentration, memory, and logical thinking. Work-related functional capacity training requires a minimum of $360 \mathrm{~min}$ of therapy.

Work-related psychological groups Work-related psychological groups are provided by a psychologist and have the purpose of supporting the concrete RTW process of each patient by focusing on personal and 
environmental factors. In the group sessions the patients are encouraged to reflect critically on their current job situations and past work-related behaviors and on how they might be able to adjust themselves or the situations accordingly. Therefore, the patients learn about stress and its influence on health and work ability and are taught about diverse coping strategies (e.g. active coping, seeking instrumental or emotional support, positive reevaluation). Group work is used to deepen the newly acquired knowledge and to transfer it to individual conditions. Techniques for immediate stress reduction such as progressive muscle relaxation, autogenic training, and meditation are shown and practiced to support coping. Additionally, communication theories and techniques are presented to the patients focusing on interpersonal relationships and communication with colleagues and supervisors. Role plays that take up realistic or even personally experienced situations at the workplace can be used to put the theory into practice. As part of work-related psychological groups, the patients learn how to apply meta-cognitions on how to achieve their RTW aims in the best possible way. To do so, they have to develop and schedule concrete RTW plans for the time after discharge from rehabilitation, taking into consideration also potential problems and barriers, as well as strategies to tackle them. Questions to clarify are: "What?", "When?", "With whom?", "How?", and "What if?". Plans are created by considering the RTW framework that is provided as the final result of social counseling. Work-related psychological groups cover at least $240 \mathrm{~min}$ of group therapy. Additional personal counseling can be provided at the request of the patient.

Intensified social counseling Additional social counseling is carried out by a social worker in group and in individual sessions. It is aimed at the clarification of the problematic work-related situations and perspectives of WMR patients by providing them with extra information and advice on social law and services. The social worker develops the RTW framework jointly with the patient and identifies which additional measures and benefits are needed and available to achieve RTW in each specific case. Such measures and benefits cover a broad scale of possible instruments and range from modifications of the work environment (e.g. height-adjustable desks) through vocational retraining (e.g. achieving a new professional title), to wage substitutes. The social worker requests the individually indicated measures and benefits from the social or health services agencies (e.g. GPI, health insurance, integration office). If needed and wanted by the patients, the social worker also establishes contact with the employer to facilitate the transition from the rehabilitation center back to the workplace (e.g. by initiating graded RTW or modifications of the work environment).
The group sessions take at least $60 \mathrm{~min}$. The personal counseling takes at least $30 \mathrm{~min}$.

\section{Participants}

Cancer patients (ICD-10: C00-D48) aged 18 to 60 years are included in the study if they have a score of $\geq 70 \%$ on the Karnofsky Performance Status Scale [50, 51] and a preliminary positive social-medical prognosis of employability for at least $3 \mathrm{~h} /$ day within the next 6 months as assessed by the rehabilitation physician in the initial examination after starting the rehabilitation program. Moreover, patients are only eligible to participate if they have an elevated risk of not returning to work. This risk is assessed by the Screening Instrument Work and Occupation (German: Screening-Instrument Beruf und Arbeit in der Rehabilitation, SIBAR) [5254]. This screening consists of three subscales. The first subscale is a 9-item scale and assesses the risk of health-related early retirement on the basis of a weighted sum score of several risk indicators (e.g. sick leave duration and the patients' belief of time to return to work). A score of more than 7 out of 19 possible points indicates an elevated risk of early retirement and thus a need for a WMR program. The second subscale is a 1-item score and assesses the amount of perceived job strain. The third scale is also a 1-item score and assesses the subjective need for work-related therapies during the rehabilitation program. If patients indicate a very stressful job situation or assume that work-related therapies will be very helpful, this is also taken as a marker of the need for a WMR program. In brief, patients are included if one of the three scales indicates a need for a WMR program. Previous studies estimated that this is the case for about half of the patients. Moreover, about a quarter of the patients has an elevated risk of early retirement based on the first scale of the SIBAR [52-54]. No exclusion criteria were defined.

\section{Randomization}

The study is designed as a cluster-randomized multicenter trial. Patients are recruited from four inpatient rehabilitation centers. Patients of a rehabilitation center who start their rehabilitation in the same week represent a cluster. These patients jointly receive either the intervention or the control treatment. Cluster-randomization was chosen to avoid spillover effects between therapy groups. Each of the four rehabilitation centers receives its own randomization schedule. The randomization lists are created by the last author using computergenerated random numbers and blocks of 4 . The administrative employees who assign the patients to their date of rehabilitation are blinded to the randomization schedule. 


\section{Sample size calculation}

Sample size calculation was done to detect a standardized mean difference (SMD) of SMD $=0.3$ in the primary outcome with $80 \%$ power and a two-sided alpha error of $5 \%$. Using a t-test, a sample size of 352 participants would be needed. The planned regression modeling of treatment effects, which includes the baseline measurement of the primary outcome as a covariate, needs a sample size that is reduced by the factor $1-\mathrm{r}^{2}$ [55]. The correlation $\mathrm{r}$ represents the correlation between baseline and follow-up measurement. The approximation of the sample size based on a t-test represents a conservative proxy, even when considering the design effects induced by the cluster-randomization or missing values. Based on expected sample attrition due to nonresponse to the 1-year follow-up questionnaire of $30 \%$, we aim to recruit at least 504 patients.

\section{Outcome measures}

Primary and secondary outcomes are assessed by questionnaires at the beginning of the inpatient rehabilitation program, at discharge from the rehabilitation program, and 3 and 12 months after discharge. Questionnaires at the beginning and discharge of the program are delivered and collected by study nurses in the rehabilitation centers. These nurses are not blinded to the treatment allocation.
Questionnaires at 3- and 12-month follow-up are sent by mail to the first author who is also not blinded to the treatment allocation. Table 3 shows all variables and their corresponding measurement point.

In the case of nonresponse to the follow-up questionnaires at 3 and 12 months after discharge, participants receive three additional reminders. The first reminder consists of a letter and is sent one week after the initial follow-up questionnaire; the second reminder includes the questionnaire again and is sent three weeks after the initial follow-up questionnaire; the third reminder also includes the questionnaire again and is sent six weeks after the initial follow-up questionnaire.

\section{Main study outcome}

The primary outcome of the study is the role functioning scale of the 30-item quality-of-life questionnaire of the European Organization for Research and Treatment of Cancer (EORTC QLQ-30). The role functioning scale comprises two items that relate to a) work and other daily activities and b) hobbies and leisure time activities. Response categories are "not at all," "a little," "quite a bit," and "very much." Single-item scores will be averaged and transformed to a score from 0 to 100 points. Higher scores represent better functioning [56].

Table 3 Time schedule of assessments and instruments

\begin{tabular}{|c|c|c|c|c|}
\hline & Admission & Discharge & 3-month follow-up & 12-month follow-up \\
\hline Role functioning (EORTC QLQ-C30) [56] & $x$ & & $x$ & $x$ \\
\hline Physical functioning (EORTC QLQ-C30) [56] & $x$ & $x$ & $x$ & $x$ \\
\hline Emotional functioning (EORTC QLQ-C30) [56] & $x$ & $x$ & $x$ & $x$ \\
\hline Social functioning (EORTC QLQ-C30) [56] & $x$ & & $x$ & $x$ \\
\hline Pain (EORTC QLQ-C30) [56] & $x$ & $x$ & $x$ & $x$ \\
\hline Global health (EORTC QLQ-C30) [56] & $x$ & $x$ & $x$ & $x$ \\
\hline Fatigue (EORTC QLQ-FA13) & $x$ & $x$ & $x$ & $x$ \\
\hline Coping with illness (FCQI) [60] & $x$ & $x$ & $x$ & $x$ \\
\hline Implementation of work-related therapies [63] & & $x$ & & \\
\hline Consistency of work-related rehabilitation strategy [63] & & $x$ & & \\
\hline Benefit from work-related therapies [63] & & $x$ & & \\
\hline Work Ability Score [59] & $x$ & $x$ & $x$ & $x$ \\
\hline Employment status & $x$ & & $x$ & $x$ \\
\hline Time of return to work & & & $x$ & $x$ \\
\hline Sick leave duration & $x$ & & $x$ & $x$ \\
\hline Disability days during the last 3 months [61] & $x$ & & $x$ & $x$ \\
\hline Patient satisfaction [64] & & $x$ & & \\
\hline Sociodemographic data & $x$ & & & \\
\hline Discrete choice experiment & $x$ & & & \\
\hline Screening Instrument Work and Occupation [67] & $x$ & & & \\
\hline
\end{tabular}

EORTC QLQ-30 30-item quality-of-life questionnaire of the European Organization for Research and Treatment of Cancer; EORTC QLQ-FA13 13-item fatigue questionnaire of the European Organization for Research and Treatment of Cancer; FCQI Freiburg Questionnaire of Coping with Illness 


\section{Secondary study outcome}

Secondary outcome measures are additional scales of the EORTC QLQ-30. These scales measure physical functioning, emotional functioning, social functioning, pain, and global health [56, 57]. Additionally, we use the 13-item fatigue module (EORTC QLQ-FA13) [58]. All scales range from 0 to 100 points. Higher scores indicate a better healthrelated quality of life.

Self-rated work ability is measured with the Work Ability Score. Ten points represent the best work ability ever achieved; 0 points indicate total work disability [59].

Disease coping is measured with three scales of the Freiburg Questionnaire of Coping with Illness (FCQI; depressive coping, active coping, distraction, and self-encouragement) with 5-point scaled items [60]. Raw item scores will be added up to a sum score for each scale. These will be used to calculate a mean scale score, resulting in values ranging from 1 to 5 points [60].

Participation in working life is measured with an assessment of the current employment status, current sick leave status, and disability days during the last 3 months $[61,62]$. The assessment after 12 months also includes a question regarding the date of initial return to work.

The realization of work-related goals and therapies during rehabilitation is assessed with a slightly modified version of a previously used set of items from a study that investigated the implementation of the WMR guidelines [63]. Participants report on 12 dichotomized items whether they received WMR contents throughout their rehabilitation program. Scores are aggregated to a total score ranging from 0 to 12 points. This score reflects the implementation of the work-related therapies. Additionally, 6 items assess the perceived diagnostic and therapeutic focus on issues of return to work and work ability, e.g. the experience of a consistent RTW strategy. These items are 5-point scaled. Scores will be summed to a total score ranging from 0 to 24 points. Finally, the subjective work-related benefit from participating in the rehabilitation program is assessed with 8 items using 5 -point scales. Scores are aggregated to a total score ranging from 0 to 32 points.

Treatment satisfaction is assessed using the German version of the Client Satisfaction Questionnaire (CSQ-8) [64]. This questionnaire uses 8 items to assess various aspects of the patient's satisfaction with the treatment. Items are 4point scaled. The sum score ranges from 8 to 32 points.

\section{Supplemental data}

The sociodemographic data that are assessed include age, sex, employment status, native language, partnership, number of children, educational level, and job qualification. Additionally, a discrete choice experiment is attached to the baseline questionnaire to assess patients' treatment preferences [65].

\section{Data management}

Questionnaires will be scanned and verified by an electronic data capture system and exported to statistical software packages for further analysis. Scanning and verifying is done by trained research assistants. Research assistants check electronically processed data item by item and compare imported data with the original questionnaire data.

If available, standard syntaxes are used to calculate scales (e.g. symptom scales of the EORTC QLQ-30) for further analyses. Each data set is checked for errors or inconsistencies before merging them with data from the other data sources or time points via the assigned study number to create a comprehensive data set. Data access is limited to the authors and the research assistants of the research team at the University of Lübeck.

\section{Statistical analysis}

Treatment effects are tested using regression analyses. In the case of binary study outcomes, logistic regression will be used (e.g. effects on employment status or sick leave at follow-up). For analysis of time to return to work, we will use proportional hazard models. Baseline parameters will be considered as covariates [66]. Due to the cluster-randomization, the induced correlation of error terms for patients with the same arrival week will be taken into account by a random effect [67-69]. Rehabilitation centers will be considered using a fixed effect.

To calculate a SMD of the between-group difference, the unstandardized regression coefficient estimate of the treatment effect will be used [70]. This estimate will be standardized using the pooled standard deviation of the observed follow-up scores [71]. In the case of binary study outcomes, estimated odds ratios will be transformed into SMDs using a logit-transformation [71, 72]. SMDs will be interpreted as suggested by Cohen [73]: small effect: $\mathrm{SMD} \geq 0.2$, medium effect: $\mathrm{SMD} \geq 0.5$, large effect: $\mathrm{SMD} \geq 0.8$.

Furthermore, to test whether the treatment effect on the primary outcome is moderated by other variables we will include two-way interaction terms in the regression model. Potential moderators that will be tested are the remaining EORTC QLQ-30 scales, fatigue, the Work Ability Score, disease coping, and the type of rehabilitation (rehabilitation following primary cancer treatment vs. follow-up rehabilitation) as well as sociodemographic variables. Continuous moderators will be standardized using a z-transformation. The parameter estimate of the interaction between the z-standardized continuous score and the treatment indicator then represents the additional treatment effects when increasing the potential moderator by about one standard deviation [74-76]. Patients will be included in our analyses if they have completed the corresponding follow-up questionnaire and will be analyzed as randomized, i.e. as intended to treat [77]. Differences will 
be regarded as significant if the two-sided p-value of a test is less than 0.05 .

Missing observations are excluded from the primary analysis. Supplementary analyses will impute missing observation by using the last observation carried forward method, in which the last observed value is used in place of the missing endpoint. No stopping guidelines are applied and no adverse health events are expected.

\section{Discussion}

Due to the increasing survival rates of cancer patients, the specific role of RTW in coping with the disease, and the higher risk of being excluded from the labor market, a clear need for interventions that support work-related outcomes can be identified. The study will provide highly ranked evidence for or against the effectiveness of WMR in cancer patients when compared to conventional MR. Positive results in favor of WMR might be expected considering the already existing body of evidence regarding WMR for other health conditions [40-45]. The current study addresses the shortcomings of previous studies by applying a randomized controlled study design and ensuring implementation of the WMR programs according to current guidelines in all participating centers. A positive evaluation of WMR in cancer patients might support the dissemination of WMR programs in German cancer rehabilitation.

\section{Trial status}

The implementation of the work-related medical rehabilitation program in all participating rehabilitation centers is finished. Patient recruitment started in June 2015 and is ongoing. For regular updates, please check the trial register entry. Items from the World Health Organization trial registration data set are presented in Additional file 1.

\section{Additional files}

Additional file 1: Table S1. World Health Organization trial registration data. (DOCX $19 \mathrm{~kb})$

Additional file 2: SPIRIT 2013 Checklist. (DOC 123 kb)

Additional file 3: Translated study information for rehabilitation patients. (DOCX $53 \mathrm{~kb}$ )

\footnotetext{
Abbreviations

CSQ, Client Satisfaction Questionnaire; EORTC QLQ-30, 30-item quality-of-life questionnaire of the European Organization for Research and Treatment of Cancer; EORTC QLQ-FA13, 13-item fatigue questionnaire of the European Organization for Research and Treatment of Cancer; FCQI, Freiburg Questionnaire of Coping with IIIness; GPI, German Pension Insurance; ICD, International Statistical Classification of Diseases and Related Health Problems; MR, medical rehabilitation; RTW, return to work; SIBAR, Screening Instrument Work and Occupation (German: Screening-Instrument Beruf und Arbeit in der Rehabilitation); SMD, standardized mean difference; SPIRIT, Standard Protocol Items: Recommendations for Interventional Trials; WMR, work-related medical rehabilitation
}

\section{Acknowledgements}

We would like to thank the Klinik Bavaria in Freyung, the Paracelsus-Klinik am See in Bad Gandersheim, the MediClin Rose Klinik Horn-Bad Meinberg, and the Röpersbergklinik in Ratzeburg for their support. This study protocol was written according to the Standard Protocol Items: Recommendations for Interventional Trials (SPIRIT) statement. The SPIRIT checklist is presented in Additional file 2.

\section{Funding}

This research is funded by the Federal German Pension Insurance for the study period from 01/2015 until 12/2017. Funding includes personnel (i.e. research associate, research assistant and study nurses) and non-personnel costs (i.e. print and travel). No additional funding can be reported. The Federal German Pension Insurance is not involved in any study activities (e.g. data analyses and interpretation). Contact at the Federal German Pension Insurance is Dr. Marco Streibelt, Department of Rehabilitation Coordination and Development of Medical and Vocational Rehabilitation, Hohenzollerndamm 47, D-10704 Berlin.

\section{Availability of data and material}

Anonymized data can be provided on request once the project ended (i.e. after submission of the final report to the funder).

\section{Authors' contributions}

$\mathrm{MB}$ and $\mathrm{BS}$ planned and designed the study. $\mathrm{MB}$ is the principal investigator and raised funding for the study. $\mathrm{MB}, \mathrm{BS}$, and JW supported the implementation in the rehabilitation centers and organized the recruitment procedure. JW and MB are conducting the statistical analyses. MB, BS, and JW drafted the manuscript. All authors read and approved the final manuscript.

\section{Competing interests}

The authors declare that they have no competing interests.

\section{Consent for publication}

This manuscript does not contain any individual person's data; hence, no consent for publication is needed.

\section{Ethical approval and consent to participate}

The study protocol was approved by the ethics committee of the University of Lübeck (14-289) and the data protection commissioner of the Federal German Pension Insurance. Approval is valid for the four participating rehabilitation centers: Klinik Bavaria in Freyung, Paracelsus-Klinik am See in Bad Gandersheim, MediClin Rose Klinik Horn-Bad Meinberg, and Röpersbergklinik in Ratzeburg. All participating rehabilitation centers are located in Germany. The trial is registered in the German Clinical Trials Register (DRKS00007770; date of registration: 05/13/2015). A translation of the study information is presented in Additional file 3.

\section{Dissemination policy}

It is planned to publish the results according the following publication plan. Interim reports will be provided to the funding organization in March 2016 and 2017. Besides publication of the study protocol, journal papers will report on the implementation phase (submission planned: July 2016), short-term results (submission planned: January 2017), the discrete choice experiment (submission planned: January 2017), 3-month follow up (submission planned: May 2017) and 12-month follow-up (submission planned: December 2017). Authorships will be handled following the standards of the International Committee of Medical Journal Editors (http://www.icmje.org/recommendations/browse/roles-and-responsibilities/ defining-the-role-of-authors-and-contributors.html).

Received: 14 January 2016 Accepted: 14 July 2016

Published online: 27 July 2016

\section{References}

1. World Health Organization. World Cancer Report 2014. Lyon: World Health Organization; 2014.

2. Robert Koch Institute and the Association of Population-based Cancer Registries in Germany. Cancer in Germany 2009/2010. 9th ed. Berlin: Robert Koch Institute; 2014 
3. Allemani C, Weir HK, Carreira H, Harewood R, Spika D, Wang X-S, et al. Global surveillance of cancer survival 1995-2009: analysis of individual data for 25676887 patients from 279 population-based registries in 67 countries (CONCORD-2). Lancet. 2015;385(9972):977-1010.

4. Petersson L-M, Wennman-Larsen A, Nilsson M, Olsson M, Alexanderson K. Work situation and sickness absence in the initial period after breast cancer surgery. Acta Oncol. 2011;50(2):282-8.

5. Paul Kl, Batinic B. The need for work: Jahoda's latent functions of employment in a representative sample of the German population. J Organ Behav. 2010;31(1):45-64.

6. Chirikos TN, Russell-Jacobs A, Cantor AB. Indirect economic effects of long term breast cancer survival. Cancer Pract. 2002;10(5):248-55

7. Griep Y, Kinnunen U, Nätti J, De Cuyper N, Mauno S, Mäkikangas A, et al. The effects of unemployment and perceived job insecurity: a comparison of their association with psychological and somatic complaints, self-rated health and life satisfaction. Int Arch Occup Environ Health. 2016;89(1):147-62.

8. Anderson NB, Armstead CA. Toward understanding the association of socioeconomic-status and health - a new challenge for the biopsychosocial approach. Psychosom Med. 1995;57(3):213-25.

9. Wolfenden B, Grace M. Returning to work after stroke: a review. Int J Rehabil Res. 2009;32(2):93-7.

10. Alaszewski A, Alaszewski H, Potter J, Penhale B. Working after a stroke: survivors' experiences and perceptions of barriers to and facilitators of the return to paid employment. Disabil Rehabil. 2007;29(24):1858-69.

11. Provencher HL, Gregg R, Mead S, Mueser KT. The role of work in the recovery of persons with psychiatric disabilities. Psychiatr Rehabil J. 2002;26(2):132-44.

12. Strober LB, Christodoulou C, Benedict RH, Westervelt HJ, Melville P, Scherl WF, et al. Unemployment in multiple sclerosis: the contribution of personality and disease. Mult Scler. 2012;18(5):647-53.

13. Rasmussen DM, Elverdam B. The meaning of work and working life after cancer: an interview study. Psychooncology. 2008;17(12):1232-8.

14. Kennedy F, Haslam C, Munir F, Pryce J. Returning to work following cancer: a qualitative exploratory study into the experience of returning to work following cancer. Eur J Cancer Care (Engl). 2007;16(1):17-25.

15. Petersson L-M, Nilsson M, Alexanderson $K$, Olsson M, Wennman-Larsen A. How do women value work shortly after breast cancer surgery and are their valuations associated with being on sick leave? J Occup Rehabil. 2013;23(3):391-9.

16. Nilsson Ml, Olsson M, Wennman-Larsen A, Petersson LM, Alexanderson K. Women's reflections and actions regarding working after breast cancer surgery - a focus group study. Psychooncology. 2013;22(7):1639-44.

17. Amir Z, Brocky J. Cancer survivorship and employment: epidemiology. Occup Med (Lond). 2009;59(6):373-7.

18. de Boer AG, Taskila T, Ojajarvi A, van Dijk FJ, Verbeek JH. Cancer survivors and unemployment: a meta-analysis and meta-regression. Jama. 2009;301(7):753-62

19. de Boer AGEM, Taskila T, Tamminga SJ, Frings-Dresen MHW, Feuerstein M, Verbeek JH. Interventions to enhance return-to-work for cancer patients. Cochrane Database Syst Rev. 2011;2:CD007569.

20. Feuerstein M, Todd B, Moskowitz M, Bruns G, Stoler M, Nassif T, et al. Work in cancer survivors: a model for practice and research. J Cancer Surviv. 2010;4(4):415-37.

21. Mehnert A. Employment and work-related issues in cancer survivors. Crit Rev Oncol Hematol. 2011;77(2):109-30.

22. Mehnert A, de Boer A, Feuerstein M. Employment challenges for cancer survivors. Cancer. 2013;119:2151-9.

23. Nilsson S, Ekberg K. Employability and work ability: returning to the labour market after long-term absence. Work. 2013;44(4):449-57.

24. Hoefsmit N, Houkes I, Nijhuis FN. Intervention characteristics that facilitate return to work after sickness absence: a systematic literature review. J Occup Rehabil. 2012;22(4):462-77.

25. Hoving $J$, Broekhuizen MLA, Frings-Dresen MHW. Return to work of breast cancer survivors: a systematic review of intervention studies. BMC Cancer. 2009;9:117.

26. Deutsche Rentenversicherung Bund. Reha-Bericht Update 2014: Die medzinische und berufliche Rehabilitation der Rentenversicherung im Licht der Statistik. Berlin: Deutsche Rentenversicherung Bund; 2014.

27. Streibelt M, Brünger M. How many work-related therapeutic services do patients with severe restrictions of work ability receive? Analysis of a representative rehabilitation sample across indications. Rehabilitation. 2014;53(6):369-75
28. Bethge M. Success factors of work-related orthopaedic rehabilitation. Rehabilitation. 2011;50(3):145-51.

29. Haaf HG. Findings on the effectiveness of rehabilitation. Rehabilitation 2005;44(05):e1-e20.

30. Hartmann U, Ring C, Reuss-Borst M. Improvement of health-related quality of life in breast cancer patients by inpatient rehabilitation. Med Klin (Munich). 2004;99(8):422-9.

31. Teichmann JV. Oncological rehabilitation: evaluation of the efficiency of inpatient rehabilitation. Rehabilitation. 2002;41(1):53-63.

32. Weis J, Moser MT, Bartsch HH. Goal-oriented evaluation of inpatient rehabilitation programs for women with breast cancer (ZESOR-study). In: Jäckel WH, Bengel J, Herdt J, editors. Research in rehabilitation: results from a research network in Southwest Germany. Stuttgart: Schattauer; 2006. p. 162-71.

33. Hartmann U, Kluge A, Ring C. Improvement of anxiety and depression in women with breast cancer during inpatient oncological rehabilitation results of a prospective study. Rehabilitation. 2006:45(2):88-94.

34. Streibelt M, Bethge M. Prospective cohort analysis of the predictive validity of a screening instrument for severe restrictions of work ability in patients with musculoskeletal disorders. Am J Phys Med Rehabil. 2015; 94(8):617-26

35. Lukasczik M, Wolf H-D, Gerlich C, Löffler S, Vogel H, Faller H, et al. Current state of vocationally oriented medical rehabilitation - a German perspective. Disabil Rehabil. 2011;33(25-26):2646-55.

36. Weis J, Moser MT, Bartsch HH. Zielorientierte Evaluation stationärer onkologischer Rehabilitationsmaßnahmen (ZESOR-Studie). Freiburg im Breisgau: Klinik für Tumorbiologie, Institut für Reha-Forschung und Prävention; 2002.

37. Streibelt M, Buschmann-Steinhage R. A profile of requirements for the performance of work related medical rehabilitation from the perspective of the statutory pension insurance. Rehabilitation. 2011;50(3):160-7.

38. Isernhagen SJ. Functional capacity evaluation: rationale, procedure, utility of the kinesiophysical approach. J Occup Rehabil. 1992;2(3):157-68.

39. Bieniek S, Bethge M. The reliability of WorkWell systems functional capacity evaluation: a systematic review. BMC Musculoskelet Disord. 2014;15:106.

40. Bethge $M$, Herbold D, Trowitzsch L, Jacobi C. Work status and health-related quality of life following multimodal work hardening: a cluster randomised trial. J Back Musculoskelet Rehabil. 2011:24(3):161-72.

41. Streibelt $M$, Bethge $M$. Effects of intensified work-related multidisciplinary rehabilitation on occupational participation: a randomized-controlled trial in patients with chronic musculoskeletal disorders. Int J Rehabil Res. 2014;37(1):61-6.

42. Kittel J, Karoff M. Improvement of worklife participation through vocationally oriented cardiac rehabilitation? Findings of a randomized control group study. Rehabilitation. 2008;47(1):14-22.

43. Streibelt M, Menzel-Begemann A. Does the success of work-related interventions in the rehabilitation of neurological diseases depend on the return-to-work prognosis? A re-analysis of 2 randomised controlled trials. Rehabilitation. 2015;54(4):252-8.

44. Koch S, Geissner E, Hillert A. Job-related effects in psychosomatic inpatient treatment: specific contributions of a cognitive-behavioural group intervention. Z Psychiatr Psych Ps. 2007:55(2):97-109.

45. Beutel ME, Zwerenz R, Bleichner F, Vorndran A, Gustson D, Knickenberg RJ. Vocational training integrated into inpatient psychosomatic rehabilitation short and long-term results from a controlled study. Disabil Rehabil. 2005;27(15):891-900.

46. Böttcher HM, Steimann M, Ullrich A, Rotsch M, Zurborn KH, Koch U, et al. Evaluation of a vocationally oriented concept within inpatient oncological rehabilitation. Rehabilitation. 2013:52(5):329-36.

47. World Health Organization. International Classification of Functioning, Disability and Health. Geneva: World Health Organization; 2001.

48. Escorpizo R, Finger M, Glässel A, Gradinger F, Lückenkemper M, Cieza A. A systematic review of functioning in vocational rehabilitation using the International Classification of Functioning, Disability and Health. J Occup Rehabil. 2011;21(2):134-46.

49. Staal JB, Hlobil H, Twisk JW, Smid T, Koke AJ, van Mechelen W. Graded activity for low back pain in occupational health care: a randomized, controlled trial. Ann Intern Med. 2004;140(2):77-84.

50. Karnofsky DA, Burchenal JH. The clinical evaluation of chemotherapeutic agents in cancer. In: MacLeod CM, editor. Evaluation of chemotherapeutic agents. New York: Columbia University Press; 1949. p. 191-205. 
51. OToole DM, Golden AM. Evaluating cancer patients for rehabilitation potential. West J Med. 1991;155(4):384-7.

52. Bürger W, Deck R. SIBAR - a short screening instrument for the assessment of need for occupation related treatment in medical rehabilitation. Rehabilitation. 2009;48(4):211-21.

53. Böttcher HM, Steimann M, Rotsch M, Zurborn K-H, Koch U, Bergelt C. Occupational stress and its association with early retirement and subjective need for occupational rehabilitation in cancer patients. Psychooncology. 2013;22(8):1807-14.

54. Böttcher HM, Steimann M, Ullrich A, Rotsch M, Zurborn K-H, Koch U, et al. Work-related predictors of not returning to work after inpatient rehabilitation in cancer patients. Acta Oncol. 2013;52(6):1067-75

55. Borm GF, Fransen J, Lemmens WAJG. A simple sample size formula for analysis of covariance in randomized clinical trials. J Clin Epidemiol. 2007:60(12):1234-8.

56. Aaronson NK, Ahmedzai S, Bergman B, Bullinger M, Cull A, Duez NJ, et al. The European Organization for Research and Treatment of Cancer QLQ-C30: a quality-of-life instrument for use in international clinical trials in oncology. J Natl Cancer Inst. 1993;85(5):365-76.

57. Damm K, Roeske N, Jacob C. Health-related quality of life questionnaires in lung cancer trials: a systematic literature review. Health Econ Rev. 2013;3(1):15

58. Weis J, Arraras Jl, Conroy T, Efficace F, Fleissner C, Görög A, et al. Development of an EORTC quality of life phase III module measuring cancer-related fatigue (EORTC QLQ-FA13). Psychooncology. 2013;22(5):1002-7.

59. IImarinen J. The Work Ability Index (WAI). Occup Med (Lond). 2007;57(2):160

60. Dörner U, Muthny FA. Comparison of two coping questionnaires in cardiological rehabilitation - the 'Trierer Skalen zur Krankheitsbewältigung' (TSK) and the 'Freiburger Fragebogen zur Krankheitsverarbeitung' (FKV). Z Med Psychol. 2008;17(2,3):125-32.

61. Klasen BW, Hallner D, Schaub C, Willburger R, Hasenbring M. Validation and reliability of the German version of the Chronic Pain Grade questionnaire in primary care back pain patients. Psychosoc Med. 2004;1:Doc07.

62. Von Korff M, Ormel J, Keefe FJ, Dworkin SF. Grading the severity of chronic pain. Pain. 1992;50(2):133-49.

63. Bethge M, Löffler S, Schwarz B, Vogel H, Schwarze M, Neuderth S. Is the guideline for work-related medical rehabilitation successfully implemented? Rehabilitation. 2014;53(3):184-90.

64. Attkisson CC, Zwick R. The client satisfaction questionnaire. Psychometric properties and correlations with service utilization and psychotherapy outcome. Eval Program Plann. 1982;5(3):233-7.

65. Bethge M. Patient preferences and willingness to wait for a work-related orthopaedic rehabilitation: a discrete choice experiment. Gesundheitswesen. 2009;71(3):152-60

66. Vickers AJ, Altman DG. Statistics notes: analysing controlled trials with baseline and follow up measurements. BMJ. 2001;323:1123-4

67. Donner A, Klar N. Design and analysis of cluster randomization trials in health research. London: Arnold; 2000.

68. Hayes RJ, Bennett S. Simple sample size calculation for cluster-randomized trials. Int J Epidemiol. 1999;28(2):319-26.

69. Hayes RJ, Alexander ND, Bennett S, Cousens SN. Design and analysis issues in cluster-randomized trials of interventions against infectious diseases. Stat Methods Med Res. 2000;9(2):95-116

70. Searle SR, Speed FM, Milliken GA. Population marginal means in the linear model: an alternative to least squares means. Am Stat. 1980;34(4):216-21.

71. Lipsey MW, Wilson DB. Practical meta-analysis. Applied Social Research Methods, vol 49. Thousand Oaks: SAGE Publications; 2000.

72. Sanchez-Meca J, Marin-Martinez F, Chacon-Moscoso S. Effect-size indices for dichotomized outcomes in meta-analysis. Psychol Methods. 2003;8(4):448-67.

73. Cohen J. A power primer. Psychol Bull. 1992;112(1):155-9.

74. Baron RM, Kenny DA. The moderator-mediator variable distinction in social psychological research: Conceptual, strategic, and statistical considerations. J Pers Soc Psychol. 1986;51(6):1173-82.

75. Brookes ST, Whitely E, Egger M, Smith GD, Mulheran PA, Peters TJ. Subgroup analyses in randomized trials: risks of subgroup-specific analyses, power and sample size for the interaction test. J Clin Epidemiol. 2004;57(3):229-36.

76. Smeets RJEM, Maher CG, Nicholas MK, Refshauge KM, Herbert RD. Do psychological characteristics predict response to exercise and advice for subacute low back pain? Arthritis Rheum. 2009;61(9):1202-9.

77. Altman DG, Schulz KF, Moher D, Egger M, Davidoff F, Elbourne D, et al. The revised CONSORT statement for reporting randomized trials: explanation and elaboration. Ann Intern Med. 2001;134(8):663-94.

\section{Submit your next manuscript to BioMed Central and we will help you at every step:}

- We accept pre-submission inquiries

- Our selector tool helps you to find the most relevant journal

- We provide round the clock customer support

- Convenient online submission

- Thorough peer review

- Inclusion in PubMed and all major indexing services

- Maximum visibility for your research

Submit your manuscript at www.biomedcentral.com/submit
C Biomed Central 